\title{
Heuristic guidelines to support conceptual design
}

Elena Mulet, Rosario Vidal

Department of Mechanical Engineering and Construction. Universitat Jaume I Castellón, Spain.

\section{Communicating author:}

Elena Mulet

Department of Mechanical Engineering and Construction. Universitat Jaume I. 12071. Castellón. SPAIN.

E-mail: emulet@emc.uji.es

Phone: 34964728117

Fax: 34964728106

\section{Rosario Vidal}

Department of Mechanical Engineering and Construction. Universitat Jaume I. 12071 Castellón. SPAIN.

E-mail: vidal@emc.uji.es.

Phone: 349647281 15. Fax number: 34964728106 


\title{
HEURISTIC GUIDELINES TO SUPPORT CONCEPTUAL DESIGN
}

\begin{abstract}
The creativity or unforeseeable nature of the design process, especially during the conceptual phase, makes it difficult to apply current design methodologies to aid designers and help them to reach a conceptual solution in an effective manner. In this paper we present some guidelines, based on experimental data about the evolution of the design process, with which to improve the effectiveness of the conceptual phase, above all as regards finding solutions. Here, effectiveness is taken as meaning quickly reaching a design solution that satisfies the requirements of the problem. Some of the guidelines obtained allow the effectiveness of a designer or a group of designers to be enhanced and are oriented towards controlling the divergence and convergence of the design space. Some guidelines for the development of interactive computer systems to aid designers are also put forward.
\end{abstract}

Keywords: design experiment, conceptual design, idea generation, function, requirement, creative methods

\author{
Abreviations \\ FBS: function, behaviour, structure \\ I/IG: global ideas ratio \\ LD: link density
}

\section{INTRODUCTION}

Obtaining a new design involves a search for solutions that places a constant demand on the designer's thinking capabilities, a demand that varies in intensity depending on the complexity of the design. Design theories and the methodologies and techniques deriving from them help designers to solve a design problem. However, despite the excellent results obtained by designers, most design tasks are still carried out by trial and error or by adopting the easiest or the most obvious solutions due to the pressure of the market and limited resources.

One of the most important characteristics of the design process is that it is a creative or unpredictable activity, especially during the conceptual phase. A creative process is understood as that which incorporates new unknown variables or knowledge that are not previously available [1]. The creative process is also opportunisc, that is, design activities are determined depending on the state of the design process, as it has been observed in empirical studies [2]. These particular characteristics of the design process make it a complex subject to study.

In addition, the spectacular and continuous growth in computational potential has meant that computers are now able to tackle a wider range of engineering design tasks. Computational design systems for supporting design synthesis are based on different methods to generate solutions that can be grouped in three categories [3]. One of these is combinational creativity in which new ideas arise from the unusual combination or association of familiar ideas $[4,5]$. A second type is that of exploratory creativity, which consists of applying search procedures within a defined conceptual space, as with scientific discovery models or heuristic search models [6]. Finally, transformational creativity systems include models based on evolutionary techniques and include procedures for modifying solutions [7-9].

As regards systems that implement the computational creative methods described by Boden, some research into the design process suggests the need to apply procedures that 
are linked to the three categories of creative methods simultaneously instead of applying them in an isolated way $[10,11]$.

One of the most significant characteristics of conceptual design is that the problem definition is not static; instead, it evolves during the course of the design process, in which continuous interactions take place between the solution and the definition of the problem. They are said to co-evolve because they exert a mutual influence on each other [12].

Taking the above into consideration, this research is based on the following hypotheses:

- In a creative design, and consequently unpredictable, oportunistic and constructive process, there are certain guidelines or parameters related to the design process that affect the solution obtained and which can be controlled in order to reach a valid solution in a short time, or to apply fewer design steps. In other words, within the creative design process, various guidelines characterise its evolution and are therefore independent of the design object or solution. Once these guidelines have been identified, the design process will be still unpredictable, but could be partially controlled through these guidelines to ease the process of obtaining a valid design solution.

- Experimental research into the tasks carried out by a designer or a group of designers can provide knowledge that can be applied not only to help the designer, but also to the development of computational design systems that will further assist the designer.

The main aim of the present work is to obtain new knowledge about the design process in order to improve creative design, both by individuals and groups, and by computational design support systems. Its specific objectives are:

- To observe how the evolution of the problem definition affects effectiveness.

- To connect experimental results and effectiveness to computational synthesis methods.

In drawing up the hypotheses of this study, the different approaches to supporting the early stages of design must be taken into consideration. Authors such as Boden defend the idea that creativity is of such an unpredictable nature that being able to offer a detailed explanation of the processes that give rise to it and the ability to foresee it are both, in general terms, impossible [3]. A similar argument is put forward by Candy, who agrees that creativity is highly unpredictable. The same author also states that the generation of new concepts by the designer, which may include for example new objects deriving from earlier ones, cannot be handled easily by computer systems $[13,14]$.

Parmee examines two approaches to design support. One focuses on the machinebased evolution of the design space relating to solutions identified from appropriate search techniques, while the other maintains that computational techniques offer their greatest potential when they are integrated with human processes, and therefore designer and machine interact $[9,15]$. The computational algorithms would be responsible for proposing solutions and the designer would process this information off-line in order to redefine the problem. It would then be reintroduced back into the computer and the design space would be refined together with the computer. It would also be important for the design team to take part in evaluating and making decisions about the concepts generated by the computer as a result of the computational procedures.

We consider that at this time the next step for the design function in design support systems consists in increasing the performance of systems in which the design process is carried out interactively with the designer.

\section{METHODOLOGY}

The methodology applied in this work is based on the experimentation in the design process under controlled laboratory conditions, using the technique of design protocol analysis. Since one of the aims is to analyse how the evolution of the problem affects 
effectiveness, it is necessary to reproduce a conceptual design situation in which the design process evolves through the incorporation of new functions and requirements.

To do so, a factor was introduced in the experiment that was expected to alter the evolution of the design process, while the other factors remain constant, thus allowing comparisons to be made. In this case the means of expressing the ideas generated is chosen because it is one of the factors that exerts an influence on the design process. Three different levels were assigned: verbal or sentential, visual and objectual.

The use of visual means during the design process has been the subject of a number of studies, the results of which show them to play several different roles in the design process, such as expanding short-term memory [16]; stimulating imagination and creative synthesis [17] as well as facilitating communication and the development and evaluation of solutions [18]. Concerning the use of objectual means, some research has revealed that designing that involves thinking with the hands offers greater feedback than using memory together with visual means [19] and that it also improves communication among members of the design team [20].

The experiment involved analysing a total of 12 teams of five students. Students were studying their first year of the Technical Engineering in Industrial Design degree course and were randomly distributed in groups of five. Four groups employed verbal means, four used visual means, while the other four utilised objectual means. All the groups started out with the same initial design requirements and had one hour in which to reach solutions for the design of a drafting table that took up as little space as possible. The experiment was directed by four lecturers from Universitat Jaume I, who explained the problem to the students and gave them the instructions to be followed throughout the session. All the students had the same role during the experiment and had no further contact with the lecturers until the end of the session. Figure 1 summarises the problem used in the experiment and the three variants that were analysed.

\section{[Insert Figure 1]}

The design protocol of the experiment was analysed by using the linkography technique, which allows design effectiveness to be evaluated by analysing the links between the ideas generated. Linkography is a graphic method for evaluating the effectiveness of the design process by studying the relations that exist among the reasoning acts that take place during design [21]. This technique was later adapted to evaluate the design process by simplifying the original analysis of the linkography [22, 23].

A method to evaluate the effectiveness of design, in which both criteria to evaluate the ideas generated and criteria related to the design process are applied [24]. The ideas are evaluated according the number obtained, and how they meet the initial requirements of the problem. Ideas considered to be invalid are those related to the requirements of the problem, but that do not constitute a viable solution or do not meet the requirements. Valid ideas are those that provide some kind of solution to the problem or make a new contribution toward solving it. Finally, if the idea does not solve any of the initial requirements, it is not considered as being related to the problem.

Ideas are aggregated at a more general level known as a global idea, which is defined by one or more ideas that contribute to the same solution to the design problem. The global idea concept is related to the concept of principle solution, and each global idea gives rise to one or more design possible solutions. The links between global ideas were then studied by applying linkographic techniques, and calculating the link density as the ratio between the number of links between global ideas and the number of global ideas (eq. 1). Finally, the ratio between the number of ideas and the global ideas was also calculated (eq. 2). 


$$
\begin{aligned}
& L D_{G I}=\frac{\text { Number of links between global ideas }}{\text { Number of global ideas }} \\
& \text { Ratio } \cdot I / G I=\frac{\text { Number of ideas }}{\text { Number of global ideas }}
\end{aligned}
$$

Analysis of Variance was performed in order to statistically validate the differences between the three variants in the experiment for each of the variables that were measured. The verbal and the objectual variants are the ones that presented the most significant differences in the analyses that were carried out and so, from now on, only the results obtained with these two variants are considered.

The evolution of the definition of the problem was studied by analysing the design protocol and by examining the FBS model of the design process in the experiment. Starting from the design protocol, the various design entities of the FBS model are identified: the functions (F) describing the goals, the behaviour (B) which describe the changes of the state of the structures, and finally the structures or solutions (S) [25-27]. In the representation of the FBS model enlarging the FBS model has been considered to indicate the modifiers of the functions [28], which other authors also call restraints and wishes [29]. The FBS model is then used to analyse the evolution of the functions and the design requirements and their relation to the solutions generated. In order to complete the analyses that were performed, the results obtained by using some descriptive models of the design have also been studied.

Finally, an analysis was made of which procedures are used to generate design structures, and whether there is any relationship between them and the creative computational methods. Both the FBS model of the design process and the design protocol were analysed in order to identify procedures which provide an explanation for the generation of design structures in the experiment carried out. In the next step, the possible relation between Boden's creative computational models (exploratory, combinational and transformational) and these procedures are examined.

Once the procedures have been identified, both the FBS model and the protocol are reanalysed in order to assign each obtained structure with the corresponding procedure used to generate it. Finally, the results obtained and the use of creative methods are interrelated in order to determine whether the effectiveness of the design can be improved further by means of the methods involved in the generation of structures. It was shown that the procedures associated with creative models (exploratory, combinational and transformational), on which computational systems are based, are applied simultaneously during the formation of the design solutions [30].

As a result of this research, a set of design guidelines to be implemented in an interactive computational design support system has been obtained to improve design effectiveness. Some of the actions are carried out by the computational system while others arise from the interaction between the designer and the computer.

\section{EFFECTIVENESS EVALUATION METHOD}

Figure 2 shows the number of ideas found to be valid, invalid and unrelated to the initial requirements that were obtained for each case of the experiment, and Figure 3 shows the percentages of the average value of each kind of idea. In the objectual variant there is a high percentage of design ideas that are valid and that satisfy the initial requirements, whereas in the verbal variant a large percentage of ideas put forward during the design do not respond to the initial requirements. 


\section{[Insert Figure 2]}

The findings from the analysis of effectiveness show that there are significant differences for most of the variables studied, with an estimation error of less than $5 \%$ [31]. Table 1 shows the average number of valid, invalid and not related ideas and the results of the Analysis of Variance (ANOVA). There are significant differences in the number of valid and not related ideas between verbal and objectual means, but the difference in the number of invalid ideas is not significant from a statistical point of view.

\section{[Insert Figure 3]}

\section{[Insert Table 1]}

Figure 4 shows all the values for the link density between the global ideas and for the ratio between the number of ideas and the number of global ideas (I/IG ratio) and Table 2 shows the mean value, the standard deviation and the results of the ANOVA. The statistical analyses show that there are significant differences in the value of these parameters depending on the means employed to express the ideas and it is therefore possible to establish a relationship between these parameters and the effectiveness of the design process. The bigger the link density and the ratio between ideas and global ideas, the greater will be the number of valid ideas that answer the initial requirements of the problem.

\section{[Insert Figure 4]}

\section{[Insert Table 2]}

The following guidelines are established for designers from the results obtained:

- Generated ideas should be revised during all the process in order to stimulate the growth of new ideas related to previous ones, thus improving the lik density index and thus effectiveness.

- New ideas should develop previous global ideas instead of trying to increase their number.

A set of guidelines to improve design effectiveness can be formulated from these results for the development of the computational system:

- The system should remind the designer of the global ideas, and ask him or her to develop existing ones instead of generating new ones.

- It should show the generated global ideas to the designer in order to stimulate him or her into obtaining new ideas related to previous ones.

Since effectiveness is not only a measurement of the validity of ideas in relation to the requirements, further studies are needed to complete these results, for example, measuring the degree of novelty of the ideas generated. 


\section{DESIGN IMPROVEMENT THROUGH THE EVOLUTION OF PROBLEM DEFINITION}

In the previous section some guidelines on how to better satisfy the problem requirements have been stablished. In accordance with the model of co-evolution, the design solutions obtained are taken as the basis for a new review of the problem requirements, so that they evolve at the same rate as the solutions during the design process.

To complete the results obtained, procedures must be established with which to select and prioritise the requirements, by studying their evolution during the design, so that the effectiveness of the design process is improved. This has been studied analysing three experiment cases where verbal means were applied and three cases where objectual means were applied. Then, this section shows an analysis about the effect of the means of expression in the evolution of the design problem.

The initial definition of the problem set out in the experiment consisted of seven requirements:

- The table must take up as little space as possible when not in use.

- The cost must be kept to a minimum.

- The table must have a board that can be tilted. The adjustment of the angle of tilt can be either continuous or discrete.

- The board surface must be located at a height between 90 and $110 \mathrm{~cm}$.

- $\quad$ The drawing surface must be large enough to take A2 format paper.

- It must be steady when it is in use.

From this set of requirements, it can be deduced that the table to be designed must also be capable of fulfilling the following functions:

- Support stress.

- Support drawing surface.

- Adjust tilt angle.

- Decrease space.

The FBS model was used to represent the number of functions and design requirements that are incorporated during the design. The time spent on each function and requirement was calculated, together with the frequency with which solutions were proposed for various functions simultaneously, and how the dedication to each function overlaps in time. Finally, the design solutions generated and the number of functions attained by each one were determined.

\subsection{TIME DEDICATED TO THE FUNCTIONS AND REQUIREMENTS OF THE PROBLEM}

Figure 5 shows the percentages of the average amount of time dedicated to the requirements and functions related to the initial requirements of the problem, called initial functions; and the time spent on those that are incorporated by the subjects during the design process, called additional functions. Table 3 shows the results of the Analysis of Variance (ANOVA) for these data.

\section{[Insert Figure 5]}

[Insert Table 3]

When objects are used the initial functions and requirements are referred to more than the $60 \%$ of the time and it is significantly greater than in the verbal case. In the verbal case, however, the time devoted to requirements and functions introduced during the design process is greater than that spent on those that were established at the outset. The 
difference in the time dedicated to the additional requirements it is not so significant from the statistical point of view.

In Table 4 the initial functions and requirements, which will be presented below in several figures, are detailed and arranged in numerical order.

\section{[Insert Table 4]}

If the time dedicated to each of the 7 initial functions and requirements is analysed separately, significant differences depending on the means of expression are observed in only three of the initial functions, as can be seen in Figure 6. These three functions are those of decreasing space, adjusting tilt angle and support stress, which are the functions that are seen as being essential in this design problem. Table 5 shows the average time and the results of the ANOVA of these functions, which is greater when objectual means are used. For function and requirement 1, 5, 6 and 7 there are no significant difference from a statistical point of view.

\section{[Insert Figure 6]}

\section{[Insert Table 5]}

One reason that accounts for why this happens is that the use of small pieces is a means of expression that is very well suited to the representation of the design solutions that are generated for the three functions indicated above. This is because they are solutions that are difficult to represent by verbalisation or by mental representation and this leads to less time being spent on them. The aid of small pieces right from the beginning of the design process, however, makes it much easier to represent solutions concerning the tilt, decreasing space and support.

The quality of the solutions was analysed by considering the degree to which they fulfilled the initial requirements and the best results were obtained in the objectual case. In addition, this is the case where more time is dedicated to the initial requirements. Previous studies show that there is a relation between the fulfilment of the design requirements and the time devoted to them in the design process. This means that requirements that are not satisfied in the design alternatives are the ones that receive least attention during the design process [32]. These results were confirmed by our experiment.

\subsection{STUDY OF THE DEGREE OF DECOMPOSITION OF THE PROBLEM}

The time dedicated to a single function, or to the simultaneous treatment of more than one, was calculated and a distinction was made between the results for the verbal and the objectual cases in order to determine how the means employed affects the decomposition of the problem. Figure 7 shows the percentage of time devoted to none or to one or more of the functions and requirements when expression is by words and when objects are used and Table 6 shows the results of the ANOVA for these data.

Within the design protocol there are moments in which no time is being devoted to any requirement or function. In some cases this situation is due to students' taking a break, and therefore attention is not paid to the problem, while in other cases the exact reason could not be determined because they did not always express verbally what they were doing when using pieces to construct the solutions.

[Insert Figure 7] 


\section{[Insert Table 6]}

As can be seen in Figure 7, more than the $50 \%$ of the time is devoted to a single design function in the verbal case. In the objectual case, however, the time dedicated to more than one function at a time is very similar to the time dedicated to a single function. It is interesting to note the large amount of time that is not dedicated to any kind of function in the objectual case. As was commented above, this is due to the fact that while students are building the solutions there are many moments when they do not comment on what they are doing. From an statistical point of view, only the time dedicate to a single function is significantly depending on the means of expression, with more than a $90 \%$ of probability.

Lastly, a detailed qualitative analysis of the distribution of the time dedicated to each function and requirement over the time the experiment lasted is conducted, as is shown in Figures 8 and 9, which represent one of the verbal and one of the objectual cases, respectively. The design functions and requirements are situated along the $\mathrm{y}$ axis and the experiment time is represented on the $\mathrm{x}$ axis. The 7 initial requirements are ordered correlatively at the beginning of the axis as shown in Table 4. The functions and requirements that are added during the design are indicated in the upper portion of the graph. Each of the segments in white indicates a period of inactivity for that function and each segment in black is a period of dedication to that function or requirement.

In the verbal case, the periods dedicated to a function or requirement are shorter than when objectual means are employed. The total time elapsed from the start of the first time the function is taken up until the last time it is set down again is also greater in the objectual case than in the verbal one, where the average number of times a same function is dealt with throughout the design process is also higher. Furthermore, in the verbal case the design time is more spread out among the functions and requirements, whereas in the objectual case only three functions (support stress, adjusting the tilt angle and decreasing space) account for almost all the time spent and often overlap with one another. In fact these three functions are interdependent, which means that many of the solutions put forward resolve the three functions jointly, without the need to break them down.

\section{[Insert Figure 8]}

[Insert Figure 9]

On analysing Figures 8 and 9 it can be seen that more functions are introduced at the beginning in the objectual case, but very few are introduced towards the later part of the time available, which means that there is greater divergence at the beginning. In the verbal case there is a lot of divergence from the beginning until the end because a large number of functions are introduced over the whole time period.

These differences come about because of the functions carried out by using objects, since they encourage the simulation of the alternatives that are generated, which in turn helps to improve these alternatives instead of diverting the designer's attention in the search for new functions. On the other hand, one way of obtaining a wide array of ideas is to introduce a large number of functions early on in the design.

\subsection{GUIDELINES FOR DESIGN EFFECTIVENESS THROUGH THE EVOLUTION OF FUNCTIONS AND REQUIREMENTS}


The results obtained were used as the basis to propose a series of heuristic guidelines to reach a valid solution to the initial requirements more effectively. These guidelines are grouped in two categories - one for those that can be applied by the designer in order to enhance conceptual design effectiveness and one for guidelines that can be used in a computational design support system that interacts with the designer.

Guidelines for designers:

- The initial functions and their interdependence should be identified and priority should be given to resolving these interconnected functions prior to other ones.

- It is beneficial to obtain design structures that allow prioritised functions to be solved, both separately and together, without decomposing these functions.

- It is appropriate to introduce additional functions once some solutions to the initial functions have already been generated. Likewise, before increasing the number of functions during the design process, it is important to have generated some solutions to other previously proposed functions. We will always be able to go back and generate new ideas for functions that have previously had solutions proposed for them.

Guidelines for the computational system:

- Solutions should be found for the interdependent or interconnected initial functions, both for each separate function and for the whole set.

- The designer should be encouraged to solve the initial functions.

- The functions should be combined periodically from the very beginning and throughout the process and shown to the designer, asking him or her to work on them and to reject any considered to be unsuitable.

- The proposed solutions should be shown to the designer in order to encourage him or her to come up with new combinations.

- The computational system should propose further functions in addition to the initial ones and the generated solutions. The relationship between the new and the initial functions will be checked by means of an ontology of functions once they and their interrelationship have been previously defined. The computer will check the characteristics of the proposed solutions, obtaining their behaviour and functionality which will then be compared with the ontology of functions in order to detect additional functions.

- In each revision of the problem definition, new solutions will be generated and shown to the designer who will be able to choose the most promising ones and include other solutions.

\section{EXPERIMENT ANALYSIS BY MEANS OF LIU, CHAKRABARTI AND BLIGH 'S MODEL}

The analyses of the experiment show that, in order to obtain design alternatives in the shortest possible time, it is extremely important to combine the proposed structures in the most interesting alternatives so as to reduce the divergence of the problem.

The goal of conceptual design is to generate good design concepts and to do so it is very important to create a large number of concepts, since the higher that number is, the greater the probability of generating valuable beginnings to solutions will be. At the same time, the chances of obtaining a good product will also rise [33]. Yet, if a lot of concepts are generated the evaluation stage will become excessively arduous. One of the models proposed for conceptual design consists in approaching the solution by going through several levels of abstraction of the solutions and by repeating cycles of divergence and convergence. The ideas put forward at the beginning consist in vague descriptions of the solution that are only expressed in words. These ideas are then developed in the conceptual phase, allowing more concrete aspects to be defined, such as the parts that make up that design solution and more information about how it works. In this way, a more defined conceptual design is gradually achieved through successive approaches that go from a more general or abstract level to a more particular one. 
Conceptual design has a first stage that is essentially divergent and then a second phase that is convergent. It is advisable to apply convergence steps in the divergent stage, as well as expanding the number of solutions. These convergence operations consist in evaluating the aspects that can be evaluated and then making a few selections in order to avoid a very high number of solutions. Similarly, once the design is essentially convergent and the alternatives are being evaluated and selected, it also becomes possible to add, in a cyclical manner, certain concepts that expand the solution space.

In other words, the most efficient model for a computational environment consists in applying numerous divergence-convergence steps within a tendency that starts off by being divergent and ends up becoming convergent, and in which solutions are first obtained for higher levels of abstraction and later for lower levels [34].

Figure 10 offers a graphic comparison of the results from the experiment with the prescriptive model developed by Liu, Chakrabarti and Bligh. The grey lines compare the traditional model of divergence and convergence with the model put forward by Liu, Chakrabarti and Bligh, in which the number of possible solutions that are explored during the divergence stage is reduced, by applying convergence operations.

The results obtained from the experiment show how design solutions can be reached faster by reducing the divergence of the design process through the use of objects to represent ideas. The black lines show the situation observed in the experiment compared with previous models. In the verbal variant the process was divergent although there were also some convergence operations. In the objectual variant there was less divergence than in the verbal case, and it was seen to be more similar to the model put forward by Liu and colleagues [34]. It also advanced towards more concrete, or less abstract, solutions for an identical period of time than when verbal means are employed. Fewer solutions were explored but there were more combination and selection operations with respect to the verbal case.

In order to achieve higher effectiveness, as established by the conceptual design model devised by Liu, Bligh and Chakrabarti, solutions should be generated for several functions simultaneously, partial solutions should be combined from the very beginning, and a reduced number of additional functions should be incorporated [34].

[Insert Figure 10]

\section{CREATIVE COMPUTATIONAL METHODS FOR DESIGN IMPROVEMENT}

The study of the mechanisms that were applied in order to obtain solutions in the experiment enabled us to determine a relationship between the computational creative models defined by Boden and the procedures that were applied during the design process. The first conclusion that can be drawn is that these design support systems must take the three types of methods into account so as to be capable of generating solutions in a more effective manner [30].

- Exploratory method: a structure that comes up from the behaviour required to accomplish one or more functions is elicited through a searching process.

- Combinational method: a searching process that ends up in a structure to address certain behaviour from one or more functions is followed by associations to adapt that existent solution to the current design problem.

- Transformational method: structures are created through the fusion of (at least) two separate structures into a new one; or structures are generated by the addition of elements from an existing structure into the structure originated in the searching process and viceversa; or structures are built through operations that exchange elements between multiple structures to generate new ones. 
Figure 11 shows the percentages of procedures from each category that were applied in order to generate solutions during the design process; it can be seen how a distinction is made between the verbal and the objectual case. With the use of verbal means there is a high percentage of search or exploratory procedures and a slightly lower number of transformation procedures. One striking feature of the objectual variant is the high percentage of transformational procedures that were applied, i.e. over $70 \%$ of the total, and the application of a number of exploratory procedures that was far lower than that of the verbal case. In both cases few combinational procedures, which are those that are related to associations and analogies, were applied. There are no significant differences as regards the application of combinational procedures, which meant that it was not possible to determine the influence they exert on the effectiveness of the design process.

\section{[Insert Figure 11]}

A relationship can be found between the procedures applied and the design solutions obtained. Various heuristic guidelines to improve effectiveness using applied procedures are:

- Procedures associated with the three creative methods, exploratory, combinational and transformational, should be applied during the design.

- In order to obtain a solution that meets initial requirements in a shorter time period, it is expedient to apply a high percentage of transformation procedures, in detriment to the number of exploratory procedures used, since fewer design solutions are generated, but with a greater degree of resolution.

- Exploratory or combinational procedures will be applied to generate a set of initial design structures, from which transformational procedures are applied in order to converge towards the design solution more quickly. Combinational and exploratory procedures should be applied at the earlier stages and throughout the whole design process, but decreasing in intensity over time.

- Transformational procedures will be successively applied to the design solutions, initially generated by exploratory and transformational procedures, in order to increase their level of resolution.

\section{CONCLUSIONS}

This research is centred on an experimental study in which the effectiveness of the design process was analysed according to the means used to express the ideas that are generated. In this study, the design problem consists in the generation of creative ideas for a new function (decrease space) of the product, considering a small set of initial requirements, and consequently the results are valid in this context. Effectiveness is understood to mean achieving design alternatives that fulfil the initial requirements of the problem in the shortest time.

The experimental data was taken as the basis for an analysis of the evolution of the design problem according to the means of expression and a relationship was established between the evolution of the problem and effectiveness. The methodology employed consists in analysing the design protocol and, at the same time, defining the FBS model for the design process. Analysing the design protocol enabled us to identify, first, the incorporation of additional functions and requirements throughout the design process and, second, the relationship these have with the structures and with the initial functions and requirements. The FBS model allowed the evolution of the functions and requirements and their relation with the design structures to be represented visually, which makes it easier to determine the differences between the case in which verbal means are employed and when objects are used. 
This analysis evidences the fact that, for this kind of design problems, if solutions are to be obtained as quickly as possible, more time should be dedicated to the initial requirements of the problem and to combining partial solutions from the outset. Identifying the interdependence between the initial functions is needed so as to be able to give priority to resolving them before the others. It is important to generate design structures that resolve these top priority functions both separately for each function and jointly for several functions. Before new functions are proposed it is important to have put forward design structures for the previous functions of the design, although more solutions can continue to be generated later on. In addition it is wise to make sure that the proposed functions are related to the initial ones or that they are stimulated by the solutions that are generated throughout the process. The structures that are obtained must be combined with each other, discarding the ones that seem to be less suited to reducing the number of possible solutions.

The results of the experiment were linked to the model of divergence and converge put forward by Liu, Bligh and Chakrabarti, using the FBS model to make a qualitative study of the cycles of divergence and convergence that appear during the design by incorporating functions, requirements and design structures. This analysis has shown that this model is followed more closely when objects are used during the divergent stage of the design. It was also found that a less abstract conceptual solution is reached in less time and divergence of the problem is reduced from the outset.

After studying the relation between effectiveness and the evolution of the design problem, a relation between the experimental results and effectiveness, and the computational synthesis methods (exploratory, combinational and transformational) is established. To do so the design protocol was studied again in order to associate the procedures carried out by designers in generating design structures with the computational methods. The results obtained make it clear that ideas are generated in the design process using three types of methods. The possible differences as regards the use of these methods was studied in the verbal and the objectual cases, and findings showed that when objects are employed there is a predominance of the use of transformational methods, which are applied successively over a set of structures obtained by exploration. Nevertheless, when only words are used to express the ideas that are generated, greater use is made of exploratory methods. In the experiment conducted for this study, no significant differences were found when combinational methods were applied.

The results obtained concerning the computational methods enabled us to draw up a series of guidelines for generating design solutions capable of enhancing the effectiveness of the design process, for design problems in which designers have to generate creative ideas for a new function in a product. In order to obtain a solution that meets the initial requirements in a shorter time period, a high percentage of transformation procedures should be applied. This will result in fewer design solutions but with a greater degree of resolution. Exploratory procedures will be applied to generate a set of initial design structures, from which transformational procedures are applied in order to converge towards the design solution more quickly.

The results of this research can contribute to the development of highly interactive systems to support conceptual design that are effective. More specifically, one of the requirements for improving these systems is the application of heuristic approaches that help the designer to progress quickly until a solution is found [35]. The experimental results obtained allowed us to establish some guidelines which can help to reach a solution more quickly in interactive computational systems.

\section{ACKNOWLEDGEMENTS}

This research is funded by Spanish Ministry of Science and Technology project DPI2002-04357C03-01. 


\section{REFERENCES}

1. Gero JS (1996) Creativity, Emergence and Evolution in Design. Knowledge-Based Systems 9 pp 435 - 448.

2. Visser W (1996) Two Functions of Analogical Reasoning in Design:A Cognitive-Psychology Approach. Design Studies Vol.17 pp 417-434.

3. Boden M (1999) Computer Models of Creativity. In: Sternberg R (eds). Handbook of Creativity. Cambridge University Press, pp 351-372.

4. Coyne R, Newton S, Sudweeks F (1993) A Connectionist View of Creative Design Reasoning. In: Gero J, Maher M (eds). Modeling Creativity and Knowledge-Based Creative Design. Lawrence Erlbaum Associates, Hillsdale, New Jersey, pp 177 - 210.

5. Maher M, Zhao F (1993) Dynamic Associations for Creative Engineering Design. In: Gero J, Maher M (eds). Modeling Creativity and Knowledge-Based Creative Design. Lawrence Erlbaum Associates, Hillsdale, New Jersey, pp 329 - 351.

6. Campbell M, Cagan J, Kotovsky K (2003) The a-Design Approach to Managing Automated Design Synthesis. Research in Engineering Design 14 pp 12 - 24.

7. Woodbury R (1993) A Genetic Approach to Creative Design. In: Proc. Modeling Creativity and Knowledge-based Creative Design, Lawrence Erlbaum Associates, Hillsdale, New Jersey.

8. Shea K, Cagan J, Fenves S (1997) A Shape Annealing Approach to Optimal Truss Design with Dynamic Grouping of Members. ASME Journal of Mechanical Design 119 3: pp 388 - 394.

9. Parmee I, Bonham C (2000) Towards the Support of Innovative Conceptual Design through Interactive Designer/Evolutionary Computing Strategies. Artificial Intelligence for Engineering Design, Analysis and Manufacturing (AIEDAM) 14 pp 3 -16.

10. Chakrabarti A (2001) Towards Hybrid Methods for Synthesis. In: Proc. ICED’01 13th International Conference on Engineering Design, Imeche, Glasgow.

11. Mulet E, Vidal R, Bermell-García P (2003) Influences of Representations on Designing and Implications for Computer-Based Design Synthesis. In: Proc. International Conference on Engineering Design, ICED 03, Design Society, Stockholm, Sweden.

12. Maher M, Poon J (1996) Modelling Design Exploration as Co-Evolution. Microcomputers in Civil Engineering 11 3: pp 195 - 210.

13. Candy L (1997) Computers and Creativity Support: Knowledge, Visualization and Collaboration. Knowledge -Based Systems 10 1: pp 3 - 13.

14. Candy L, Edmonds E (2000) Creativity Enhancement with Emerging Technologies. Communications of the ACM 43 8: pp 62 - 65.

15. Parmee IC (2001) Evolutionary and Adaptive Computing in Engineering Design, SpringerVerlag, London.

16. Ullman D, Wood S, Craig D (1990) The Importance of Drawing in the Mechanical Design Process. Computational Graphics 14 2: pp 263 - 274.

17. Purcell A, Gero J (1998) Drawings and the Design Process. Design Studies 19 4: pp 389 - 430.

18. Schütze M, Sache P, Römer A (2003) Support Value of Sketching in the Design Process. Research in Engineering Design 14 pp 89 - 97. 
19. Candy L, Edmonds E (1996) Creativity Design of the Lotus Bicycle: Implications for Knowledge Support System Research. Design Studies 17 1: pp 71 - 90.

20. Harrison S, Minneman S (1996) A Bike in Hand: A Study of 3-D Objects in Design. In: Cross N, Christiaans H, Dorst K (eds). Analysing Design Activity. John Wiley \& Sons, Chichester, pp 417 - 436.

21. Goldschmidt G (1990) Linkography: Assessing Design Productivity. In: Proc. 10th European Meeting on Cybernetics and Systems Research, Cybernetics and systems World Scientific Pub Co, Vienna.

22. VanderLugt R (2000) Devoloping a Graphic Tool for Creative Problem Solving in Design Groups. Design Studies 21 5: pp 505-522.

23. Van der Lugt R (2003) Relating the Quality of the Idea Generation Processs to the Quality of the Resulting Ideas. In: Proc. International Conference on Engineering Design, ICED 03, Design Society, Stockholm, Sweden.

24. Vidal R, Mulet E, Gómez-Senent E (2004) Effectiveness of the Means of Expression in Creative Problem Solving in Design Groups. Journal of Engineering Design 15 3: pp 285-297.

25. Gero J (1990) Design Prototypes: A Knowledge Representation Schema for Design. AI magazine 11 4: pp 26 - 36.

26. Takeda H, Veerkamp P, Tomiyama T, Yoshikawa H (1990) Modeling Design Processes. AI Magazine 11 4: pp 37-48.

27. Umeda Y, Takeda H, Tomiyama T, Yoshikawa H (1990) Function, Behaviour, and Structure. In: Gero J (eds). Applications of Artificial Intelligence in Engineering V. 1. Springer, Berlin, pp 177-194.

28. Takeda H, Yoshioka M, Tomiyama T, Shimomura Y (1996) Analysis of Design Protocol by Functional Evolution Process Model. In: Cross N, Christiaans H, Dorst K (eds). Analysing Design Activity. pp 187-209.

29. Li Q, Zhang WJ (1998) Computer Comparison of Design Knowledge. Proceedings of the Institution of Mechanical Engineers 212 Part B: pp 635-645.

30. Mulet E, Vidal R, Bermell-García P (2003) Influences of Representations on Designing and Implications for Computer-Based Design Synthesis. In: Proc. International Conference on Engineering Design, ICED, Stokholm.

31. Vidal R, Mulet E, Gómez-Senent E (2004) Effectiveness of the Means of Expression in Creative Problem Solving in Design Groups. Journal of Engineering Design 15 3: pp

32. Nidamarthi S, Chakrabarti A, Bligh TP (2001) Improving Requirement Satisfaction Ability of the Designer. In: Proc. International Conference in Engineering Design, ICED, 1 Imeche, Glasgow, U.K.

33. Chakrabarti A, Bligh TP (1996) An Approach to Functional Synthesis of Solutions in Mechanical Conceptual Design. Part Iii: Spatial Configuration. Research in Engineering Design 2 pp 116 - 124.

34. Liu Y, Bligh T, Chakrabarti A (2003) Towards an 'Ideal' Approach for Concept Generation. Design Studies 24 pp 341 - 355.

35. Wiegers T, Horváth I, Vergeest J, Opiyo E, Kuczogi G (1999) Requierements for Highly Interactive System Interfaces to Support Conceptual Design. In: Kals H, Houten F v (eds). Integration of Process Knowledge into Design Support Systems. Kluwer Academic Publishers, Enschede, pp 69-78. 
Figure 1. Means of expression in the experiment.

Figure 2. Evaluation for idea generated at each run of the verbal and objectual experiment.

Figure 3. Evaluation of the generated ideas.

Figure 4. Link density and I/IG ratio for each experiment run.

Figure 5. Time dedicated to the initial and to the additional requirements.

Figure 6. Time devoted to each initial requirement.

Figure 7. Simultaneous dedication of time to functions and requirements.

Figure 8. Overlap between the requirements and functions in one of the verbal cases.

Figure 9. Overlap between the requirements and functions in one of the objectual cases.

Figure 10. Analysis of the experiment with different divergence-convergence models.

Figure 11. Percentages in which the different types of procedures used to obtain solutions were applied.

TABLE 1. Statistics obtained for the evaluation variables.

\begin{tabular}{|l|c|c|c|c|c|c|}
\hline & \multicolumn{2}{|c|}{ Verbal or sentential } & \multicolumn{2}{|c|}{ Objectual } & \multicolumn{2}{c|}{ Results of ANOVA } \\
\hline & $\begin{array}{c}\text { Arithmetic } \\
\text { mean }\end{array}$ & S.D. & $\begin{array}{c}\text { Arithmetic } \\
\text { mean }\end{array}$ & S.D. & $\begin{array}{c}\text { ANOVA } \\
\text { F ratio }\end{array}$ & $\begin{array}{c}\text { ANOVA } \\
\text { Mistake } \\
\text { Probability }\end{array}$ \\
\hline $\begin{array}{l}\text { Number of Valid } \\
\text { Ideas }\end{array}$ & 26 & 14 & 87 & 15 & 35.12 & $<0.001$ \\
\hline $\begin{array}{l}\text { Number of Invalid } \\
\text { /Rejected Ideas }\end{array}$ & 10 & 4 & 13 & 7 & 0.63 & 0.46 \\
\hline $\begin{array}{l}\text { Number of Not } \\
\text { Related Ideas }\end{array}$ & 78 & 9 & 7 & 8 & 133.39 & $<0.001$ \\
\hline Number of Ideas & 114 & 10 & 107 & 9 & & \\
\hline
\end{tabular}

TABLE 2. Statistics for the effectiveness of design in relation to the means of expression.

\begin{tabular}{|l|c|c|c|c|c|c|}
\hline & \multicolumn{2}{|c|}{ Verbal variant } & \multicolumn{2}{c|}{ Objectual variant } & \multicolumn{2}{c|}{ Results of ANOVA } \\
\hline $\begin{array}{l}\text { Parameter } \\
\text { measured }\end{array}$ & $\begin{array}{c}\text { Arithmetic } \\
\text { Mean }\end{array}$ & S.D. & $\begin{array}{c}\text { Arithmetic } \\
\text { Mean }\end{array}$ & S.D. & $\begin{array}{c}\text { ANOVA } \\
\text { F ratio }\end{array}$ & $\begin{array}{c}\text { ANOVA } \\
\text { Mistake } \\
\text { Probability }\end{array}$ \\
\hline $\begin{array}{l}\text { Number of } \\
\text { global ideas }\end{array}$ & 45 & 7.4 & 12 & 3.7 & 66.43 & $<0.001$ \\
\hline Ratio I/GI & 2.6 & 0.60 & 10.1 & 3.36 & 19.30 & 0.005 \\
\hline Link Density & 0.54 & 0.085 & 0.91 & 0.028 & 69.17 & $<0.001$ \\
\hline
\end{tabular}

TABLE 3. Statistics obtained for the time dedicated to the initial and the additional functions and requirements.

\begin{tabular}{|l|c|c|c|c|c|c|}
\hline & \multicolumn{2}{|c|}{ Verbal/sentential } & \multicolumn{2}{c|}{ Objectual } & \multicolumn{2}{c|}{ Results of ANOVA } \\
\hline & $\begin{array}{c}\text { Arithmetic } \\
\text { mean }\end{array}$ & S.D. & $\begin{array}{c}\text { Arithmetic } \\
\text { mean }\end{array}$ & S.D. & $\begin{array}{c}\text { ANOVA } \\
\text { F ratio }\end{array}$ & $\begin{array}{c}\text { ANOVA } \\
\text { Mistake } \\
\text { Probability }\end{array}$ \\
\hline Time for initial requirements & 21.4 & 10.1 & 62.9 & 4.0 & 43.9 & 0.03 \\
\hline
\end{tabular}




\begin{tabular}{|l|l|l|l|l|l|l|}
\hline or functions (minutes) & & & & & & \\
\hline $\begin{array}{l}\text { Time for additional } \\
\text { requirements or functions } \\
\text { (minutes) }\end{array}$ & 35.7 & 13.6 & 17.7 & 11.7 & 3.0 & 0.157 \\
\hline
\end{tabular}

TABLE 4. List of initial functions and requirements.

\begin{tabular}{|l|l|}
\hline Code & Function or requirement \\
\hline FR 1 & Minimum cost \\
\hline FR 2 & Decrease space \\
\hline FR 3 & Adjust tilt angle \\
\hline FR 4 & Support stress \\
\hline FR 5 & Steady \\
\hline FR 6 & Height between 90 and $110 \mathrm{~cm}$ \\
\hline FR 7 & Support drawing surface \\
\hline
\end{tabular}

TABLE 5. Statistics obtained for the time dedicated to the initial functions and requirements.

\begin{tabular}{|l|l|l|l|l|l|l|}
\hline & \multicolumn{2}{|l|}{ Verbal/ sentential } & \multicolumn{2}{|c|}{ Objectual } & \multicolumn{2}{c|}{ Results of ANOVA } \\
\hline & $\begin{array}{l}\text { Arithmetic } \\
\text { mean }\end{array}$ & S.D. & $\begin{array}{l}\text { Arithmetic } \\
\text { mean }\end{array}$ & S.D. & $\begin{array}{l}\text { ANOVA } \\
\text { F ratio }\end{array}$ & $\begin{array}{l}\text { ANOVA } \\
\text { Mistake } \\
\text { Probability }\end{array}$ \\
\hline Time for FR 2 & 6.2 & 3.5 & 24.2 & 6.3 & 18.69 & 0.012 \\
\hline Time for FR 3 & 3.4 & 1.9 & 11.4 & 4.3 & 8.57 & 0.043 \\
\hline Time for FR4 & 5.7 & 3.4 & 19.9 & 6.2 & 11.89 & 0.026 \\
\hline
\end{tabular}

TABLE 6. Statistics obtained for the time dedicated to one, none or more than one function and requirements.

\begin{tabular}{|l|c|c|c|c|c|c|}
\hline & \multicolumn{2}{|c|}{ Verbal/sentential } & \multicolumn{2}{|c|}{ Objectual } & \multicolumn{2}{c|}{ Results of ANOVA } \\
\hline & $\begin{array}{c}\text { Arithmetic } \\
\text { mean }\end{array}$ & S.D. & $\begin{array}{c}\text { Arithmetic } \\
\text { mean }\end{array}$ & S.D. & $\begin{array}{c}\text { ANOVA } \\
\text { F ratio }\end{array}$ & $\begin{array}{c}\text { ANOVA } \\
\text { Mistake } \\
\text { Probability }\end{array}$ \\
\hline $\begin{array}{l}\text { Time dedicated to more than } \\
\text { one function }\end{array}$ & 10.3 & 5.6 & 18.5 & 5.2 & 3.50 & 0.135 \\
\hline $\begin{array}{l}\text { Time dedicated to one } \\
\text { function }\end{array}$ & 31.3 & 6.8 & 17.9 & 7.9 & 4.91 & 0.091 \\
\hline $\begin{array}{l}\text { Time dedicated to any } \\
\text { function }\end{array}$ & 15.5 & 12.6 & 19 & 5.8 & 0.192 & 0.684 \\
\hline
\end{tabular}

